\title{
Cytokine storm and the prospects for immunotherapy with COVID-19
}

\section{ABSTRACT}

Knowledge about the pathobiology of SARS-CoV-2 as it interacts with immune defenses is limited. SARS-CoV-2 is spread by droplets that come into contact with mucous membranes. COVID-19 is characterized by 2 or 3 stages: most patients who recover experience 2 stages of illness commencing with an asymptomatic or paucisymptomatic incubation period, followed by a nonsevere symptomatic illness lasting for several weeks, occurring in about $80 \%$ of those infected. In the remainder, a third phase marked by a severe respiratory illness, often accompanied by multisystem dysfunction, coagulopathy, and shock is observed. This phase of the illness is characterized by hypercytokinemic inflammation and is often referred to as "cytokine storm." While the immunopathogenesis remains unclear, prospects of treating this severe phase of the illness with immunotherapy are evolving, with some treatments showing promise.
Dr. Calabrese has disclosed financial interests (consulting, teaching, and speaking) with AbbVie, Bristol Myers Squibb, Crescendo Biologics, GlaxoSmithKline, Genentech/Roche, Horizon Therapeutics, Janssen, Novartis, and Pfizer.

doi:10.3949/ccjm.87a.ccc008
A s we learn about COVID-19, we rec$A$ ognize that there are gaping holes in our knowledge of the pathobiology of SARS$\mathrm{CoV}-2$ as it interacts with our immune defenses. Epidemiologically, we know that most people, especially young and healthy ones, do quite well at defending themselves from this infection and that even those with severe disease tend to recover without sequelae. We also know that not everyone has a relatively benign disease course and that risk factors for progression are dominated by age and comorbidities, especially cardiovascular disease, diabetes, and obesity.

While some of these clinical findings seem to have face-validity, others are not so clear. Why is age such a dominant risk factor, but on the other hand, why do some young, otherwise seemingly healthy individuals succumb to the infection? We do not yet have complete answers to these questions.

\section{THREE STAGES OF DISEASE}

To tackle this problem, we must first examine what is known about the interaction between the pathogen and the host immune system. SARS-CoV-2 is spread by droplets that come into contact with mucous membranes. Interestingly, not all individuals who are exposed acquire the infection. Once a person is infected, the disease progresses through 2 or 3 main stages (Figure 1).

Stage 1 is an asymptomatic or paucisymptomatic incubation period in which there is a high level of viral shedding in the upper respiratory tract. Implicitly, this stage marks engagement of the innate immune system as the initial mode of host defense. 


\begin{tabular}{|c|c|c|c|}
\hline & Stage 1 & Stage 2 & Stage 3 \\
\hline & Asymptomatic & Nonsevere symptomatic & $\begin{array}{l}\text { Severe respiratory- } \\
\text { inflammatory }\end{array}$ \\
\hline $\begin{array}{l}\text { Immune response } \\
\text { over time: }\end{array}$ & $\begin{array}{l}\text { Innate immune } \\
\text { activation }\end{array}$ & $\begin{array}{l}\text { Adaptive immune } \\
\text { activation }\end{array}$ & $\begin{array}{l}\text { Cytokine release } \\
\text { syndrome }\end{array}$ \\
\hline $\begin{array}{l}\text { Self-limiting in } 80 \% \\
\text { Severe in } 15 \%-20 \% \\
\text { Fatal in } 1 \%-2 \%\end{array}$ & $\begin{array}{l}\text { Viral engagement } \\
\text { of PAMPs } \\
\text { Low type } 1 \text { IFN }\end{array}$ & $\begin{array}{l}\text { Generation of specific } \\
\text { antibodies and T-cell response } \\
\text { Release of DAMPs }\end{array}$ & $\begin{array}{l}\text { IL-1, IL-6, TNF, GM-CSF, } \\
\text { IFN-gamma, others } \\
\text { Coagulopathy } \\
\text { Complement }\end{array}$ \\
\hline
\end{tabular}

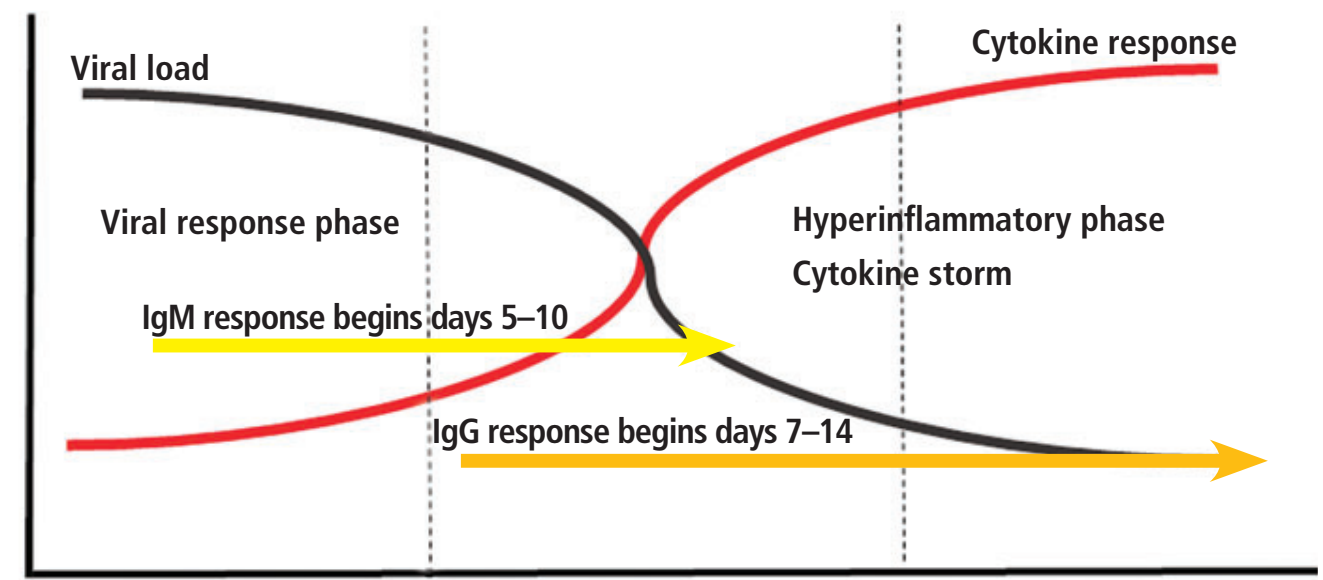

Time course

DAMPs = damage-associated molecular patterns; GM-CSF = granulocyte macrophage colony-stimulating factor; IFN = interferon; IgM = immunoglobulin M; IL-1 = interleukin 1; IL-6 = interleukin 6; PAMPs = pathogen-associated molecular patterns; TNF = tumor necrosis factor

Figure 1. Three stages of COVID-19 disease.

Stage 2 is a period of nonsevere symptomatic illness in which viral loads peak approximately 5 days after symptom onset. ${ }^{1}$ At this stage adaptive immunity is engaged, allowing development of specific $\mathrm{T}$ - and B-cell responses required to end the infectious process. The disease ends in stage 2 in approximately $80 \%$ of infected individuals.

Finally, stage 3 is characterized by severe respiratory illness with progressive pneumonitis that may or may not lead to respiratory failure, which, in its final stages causes diffuse alveolar damage. Stage 3 is also frequently attended by progressive fever, multiorgan dysfunction, hypercoagulability, and shock. ${ }^{2}$

Immunologically, SARS-CoV-2 infects cells that express the angiotensin-converting enzyme 2 receptor, including cells of the respiratory tract, endothelial cells, and likely hematopoietic cells, including macrophages. With full engagement of the integrated immune response, the development of neutraliz- ing antibodies is believed to be a critical event in recovery as well as the generation of virusspecific T-cell responses, ultimately leading to viral clearance. ${ }^{3}$

Attempts to correlate the stages of clinical disease described above with SARS-CoV-2 viral loads from respiratory secretions, blood, and tissues have yielded conflicting results. Some patients with advanced disease have high viral loads while others do not. ${ }^{4}$

\section{WHY DO SOME PATIENTS GO ON TO STAGE 3?}

Why host antiviral defenses fail and why some patients go on to stage 3 is not yet clear, but attempts to reconcile these findings suggest that this progression may be driven by ongoing viral infection. In support of this hypothesis is a recent study documenting that SARS-CoV-2 infection actually induces a low interferon response, an immune pathway critical for antiviral defense, while at the same time inducing 
a strong inflammatory response, thus creating a perfect storm of continued viral replication and unbridled inflammation. . $^{5,6}$

The clinical state of patients with stage 3 disease is characterized by hypercytokinemic inflammation. This syndrome has variably been referred to as "cytokine storm." The cytokine storm of COVID-19 bears similarities to other conditions that are also referred to under this umbrella, including primary hemophagocytic lymphohistiocytosis (HLH), as well as secondary forms such as macrophage activation syndrome (MAS) and secondary HLH, which are often encountered in the setting of autoimmunity, cancer, or viral infections. ${ }^{7,8}$ In COVID-19, and unlike in MAS or secondary HLH, the primary target organ is the lung, leading to an acute respiratory distress syndrome. While stage 3 COVID-19 is not secondary HLH or MAS, it does share features both clinically and pathologically. ${ }^{5}$

Recently, a variant of this cytokine storm has been described in children with COVID-19 and has been dubbed multisystem inflammatory syndrome in children (MIS-C). ${ }^{9}$

Laboratory features are quite similar among these disorders, with marked elevations of acute-phase reactants (eg, C-reactive protein, ferritin), lymphopenia, coagulation defects, and elevated levels of numerous inflammatory cytokines; prominent among them are interleukin 6 (IL-6), IL-1, IL-2, IL-7, IL-17, granulocyte macrophage-colony stimulating factor (GM-CSF), and tumor necrosis factor (TNF). ${ }^{10}$

Why there is an increased incidence of this inflammatory late-stage complication in select young individuals and more frequently in patients who are elderly and in those with comorbidities is poorly understood. Interestingly, though, in a study attempting to further understand why otherwise-healthy individuals can die from viral illness, $30 \%$ of patients dying from H1N1 influenza were found to carry single copies of genes commonly encountered in patients with $\mathrm{HLH},{ }^{11}$ suggesting a link between immune predisposition to HLH and outcome. We can also postulate that the chronic low-grade inflammation and an increase in self-reactivity that characterize the aging immune system also may contribute. Importantly, recent studies have shown that

\section{TABLE 1}

\section{Immunotherapeutic strategies for COVID-19}

Kinase inhibitors

Tofacitinib, baricitinib, ruxolitinib, others

Targeted therapy

Inhibitors of interleukin 1 (IL-1), IL-6, tumor necrosis factor, and granulocyte macrophage colony-stimulating factor; interferon gamma

Tolerogenic therapies

T-cell regulators, low-dose IL-2

Cellular therapies

Natural killer cell therapies, antiplasmacytoid dendritic cell, others

Passive therapy

Intravenous immune globulin, immune plasma, specific antibodies

Nonspecific therapies

Glucocorticoids, calcineurin inhibitors, mammalian target of rapamycin inhibitors

immunologic aging proceeds at different rates in different individuals; thus, mere chronologic age is, not surprisingly, a relatively crude predictor of COVID-19 progression. ${ }^{12}$

\section{IMPLICATIONS FOR THERAPY}

From a therapeutic perspective, there is a clear need for an effective antiviral agent that can prevent viral infection in exposed individuals and limit tissue damage in those with established disease (stages 1 and 2).

In stage 3 , in the absence of any effective antiviral therapy, we are relegated to supportive care. It is at this stage that the experimental use of agents designed to limit tissue damage driven by uncontrolled inflammation is being investigated. Given the similarities between stage 3 COVID-19 and other hypercytokinemic states, a variety of nonspecific immunosuppressive strategies have been proposed, such as glucocorticoids, hydroxychloroquine, colchicine, and other immunomodulators, as well as Janus kinase inhibitors and a number of targeted therapies directed at pivotal cytokines (Table 1). For now, the experience with such agents largely consists of anecdotal case reports and small clinical trials. ${ }^{13}$

As of this writing, more than 900 clinical trials of various therapeutics for COVID-19 are registered at clinicaltrials.gov. Agents that have been proposed or are in use include anti-

\section{Despite gaping holes in our knowledge, treatment is evolving}


IL-1, anti-IL-6, anti-GM-CSF, and anti-TNF drugs, and Janus kinase inhibitors. A conceptual framework of such therapies is displayed in Table 1.

\section{Anti-IL-6 and anti-IL-1 agents:} Center stage for COVID-19 stage 3 disease

Among these therapies, agents that target IL-6 have perhaps generated the greatest enthusiasm. Numerous IL-6-targeting agents are being tested in COVID-19 including those targeting the IL- 6 receptor (tocilizumab, sarilumab) and those targeting IL-6 itself (siltuximab, clazakizumab, and sirukumab). Two of these agents-tocilizumab (NCT04320615) and sarilumab (NCT04315298)—are already in advanced stages of multicenter randomized control trials, and data should be forthcoming soon.

Interest in IL-6 is strong, as it is a pleomorphic cytokine produced by both hematopoietic and viscerosomatic cells and has far-reaching effects on immune function and diverse nonimmune physiologic processes. ${ }^{14}$ It is a key upstream driver of inflammation and has been successfully targeted therapeutically. IL-6 also has been shown to be a predictor of respiratory failure.

Of particular relevance for stage 3 COVID-19 disease, targeting IL-6 with tocilizumab is now indicated for treatment of cytokine storm accompanying chimeric antigen receptor (CAR)T-cell therapy. ${ }^{15}$ Clinical support for advancing the study of IL-6 inhibition in COVID-19 has come from a variety of sources, including anecdotes from now-widespread off-label use of tocilizumab, case reports, and small series ${ }^{16}$ in which rapid reversal of laboratory and clinical parameters were reported. Balancing enthusiasm for such a strategy is the known pivotal role of IL-6 in host defense, particularly in defense against respiratory viruses. ${ }^{17}$

IL-1 is another inflammatory cytokine that could potentially be targeted to treat various cytokine storm syndromes. IL-1 is an upstream mediator of inflammation and is produced by the NLPR3 inflammasome; it has been incriminated in the pathogenesis of COVID-19, having been detected in lung tissue by a variety of techniques. ${ }^{18}$

As of this writing, 3 small nonrandomized case series have demonstrated benefit of IL-1 inhibition in COVID-19. ${ }^{18,19}$ The largest of these series, ${ }^{19}$ while suffering from the use of a retrospectively derived comparator group, demonstrated meaningful improvement in reducing the need for mechanical ventilation with the use of anakinra, a human IL-1 receptor antagonist. Anakinra has a short half-life, a large therapeutic window, and a well-established safety profile, and can be given by subcutaneous and intravenous routes. ${ }^{18}$ Large prospective trials are now under way and results are eagerly awaited.

\section{Safety concerns}

Above all, there are serious considerations regarding untoward toxicity. Paramount among safety considerations is the potential for targeted therapies to suppress the host's immune response and further limit failing antiviral defenses. Theoretically, the short-term use of such agents is likely to be less immunosuppressive than observed in long-term clinical use, but this hypothesis remains unproven. Concerns regarding the use of Janus kinase inhibitors is of particular note since they can further serve to suppress type I and III interferons, which are critical in antiviral defense. ${ }^{20}$ Also critical is the potential risk associated with the timing of therapy. Administering treatment too early may compromise antiviral immunity, while waiting too long may risk irreversible organ damage.

\section{Other novel therapies}

Other novel therapies used alone or in combination include targeting GM-CSF, granulocyte-colony stimulating factor, and Janus kinase. These have been reviewed or mentioned in several excellent narrative reviews. $5,8,13,21$ 


\section{REFERENCES}

1. To KK-W, Tsang OT-Y, Leung W-S, et al. Temporal profiles of viral load in posterior oropharyngeal saliva samples and serum antibody responses during infection by SARSCoV-2. Lancet Infect Dis 2020 May; 20(5):565-574. doi: 10.1016/S1473-3099(20)30196-1

2. Berlin DA, Gulick RM, Martinez FJ. Severe Covid-19. N Engl J Med 2020 May 27. doi:10.1056/NEJMcp2009575

3. Li G, Fan Y, Lai Y, et al. Coronavirus infections and immune responses. J Med Virol 2020; 92(4):424-432. doi:10.1002/jmv.25685

4. Joynt GM, Wu WK. Understanding COVID-19: what does viral RNA load really mean? Lancet Infect Dis 2020 Mar 27 pii: S1473-3099(20)30237-1. doi:10.1016/S1473-3099(20)30237-1

5. McGonagle D, Sharif K, O'Regan A, Bridgewood C. The role of cytokines including interleukin- 6 in COVID-19 induced pneumonia and macrophage activation syndrome-like disease. Autoimmun Rev 2020; 19(6):102537. doi:10.1016/j.aut- rev.2020.102537

6. Blanco-Melo D, Nilsson-Payant B, Liu W-C et al. Imbalanced host response to SARS-CoV-2 drives development of COVID-19. Cell 2020; May 13; S0092-8674(20)30489-X. doi:10.1016/j.cell.2020.04.026

7. Grom AA, Horne A, De Benedetti F. Macrophage activation syndrome in the era of biologic therapy. Nat Rev Rheumatol 2016; 12(5):259-268. doi:10.1038/nrrheum.2015.179

8. Henderson LA, Canna SW, Schulert GS. On the alert for cytokine storm: immunopathology in COVID-19. Arthritis Rheum 2020 April 16. doi:10.1002/art.41285

9. Jones VG, Mills M, Suarez D, et al. COVID 19 and Kawasaki disease: novel virus and novel case. Hosp Pediatr 2020; doi:10.1542/hpeds.2020-0123

10. Mehta P, McAuley DF, Brown M, et al. COVID-19: consider cytokine storm syndromes and immunosuppression. Lancet 2020; 395(10229):1033-1034. doi:10.1016/S0140-6736(20)30628-0

11. Schulert GS, Zhang $\mathbf{M}$, Fall $\mathbf{N}$, et al. Whole-exome sequencing reveals mutations in genes linked to hemophagocytic lymphohistiocytosis and macrophage activation syndrome in fatal cases of H1N1 influenza. J Infect Dis 2016; 213 (7):1180-1188. doi:10.1093/infdis/jiv550
12. Alpert A, Pickman $Y$, Leipold $M$, et al. A clinically meaningful metric of immune age derived from highdimensional longitudinal monitoring. Nat Med 2019; 25(3):487-495. doi:10.1038/s41591-019-0381-y

13. Ceribelli A, Motta F, De Santis M, et al. Recommendations for coronavirus infection in rheumatic diseases treated with biologic therapy. J Autoimmun 2020; 109:102442. doi:10.1016/j.jaut.2020.102442

14. Calabrese LH, Rose-John S. IL-6 biology: implications for clinical targeting in rheumatic disease. Nat Rev Rheumatol 2014; 10(12):720-727. doi:10.1038/nrrheum.2014.127

15. Chen $\mathrm{H}$, Wang $\mathrm{F}$, Zhang $\mathrm{P}$, et al. Management of cytokine release syndrome related to CAR-T cell therapy. Front Med 2019; 13(5):610- 617. doi:10.1007/s11684-019-0714-8

16. Zhang C, Wu Z, Li JW, Zhao H, Wang GQ. The cytokine release syndrome (CRS) of severe COVID-19 and interleukin- 6 receptor (IL-6R) antagonist tocilizumab may be the key to reduce the mortality. Int J Antimicrob Agents 2020 March 29:105954. doi:10.1016/j.ijantimi- cag.2020.105954

17. Rose-John S, Winthrop K, Calabrese L. The role of IL-6 in host defence against infections: immunobiology and clinical implications. Nat Rev Rheumatol 2017; 13(7):399 409. doi:10.1038/nrrheum.2017.83

18. Calabrese LH, Calabrese C. Cytokine release syndrome and the prospects for immunotherapy with COVID-19. Part 2: the role of IL-1. Cleve Clin J Med. In press. doi:10.3949/ccjm.87a.ccc043

19. Huet T, Beaussier H, Voisin O, et al. Anakinra for severe forms of COVID-19: a cohort study. Lancet May 29, 2020. doi:10.1016/S2665-9913(20)30164-8

20. Winthrop KL. The emerging safety profile of JAK inhibitors in rheumatic disease. Nat Rev Rheumatol 2017; 13(4):234-243. doi:10.1038/nrrheum.2017.23

21. Ascierto PA, Fox B, Urba W, et al. Insights from immunooncology: the Society for Immunotherapy of Cancer statement on access to IL-6-targeting therapies for COVID-19. J Immunother Cancer 2020 Apr; 8(1):e000878. doi:10.1136/jitc-2020-000878

Address: Leonard H. Calabrese, DO, Department of Rheumatic and Immunologic Diseases, A50, Cleveland Clinic, 9500 Euclid Avenue, Cleveland, OH 44195; calabrl@ccf.org 